\title{
Effect of different exercise trainings on $\beta$-cell function, insulin resistance, and osteocalcin levels in overweight men
}

\author{
Mehdi Rostamizadeh $^{1}{ }^{(\mathbb{D}}$, Alireza Elmieh $^{2 *}{ }^{(\mathbb{D}}$, Farhad Rahmani Nia ${ }^{3}$ \\ 'Department of Physical Education, Ahar branch, Islamic Azad university, Ahar, Iran \\ ${ }^{2}$ Department of Physical Education, Rasht branch, Islamic Azad university, Rasht, Iran \\ ${ }^{3}$ Department of Physical Education, University of Guilan, Rasht, Iran
}

*Corresponding Author: Alireza Elmieh, Department of Physical Education, Rasht Branch, Islamic Azad University, Rasht, Iran, Tel: +989111359121, Email: elmieh@iaurasht.ac.ir

\begin{abstract}
Background and aims: Many findings have shown the potential relation between osteocalcin (OCN) and regulating energy metabolism. In addition, it has been revealed that physical activity increases OCN levels. Therefore, the present study aimed to investigate the effects of different exercise trainings on $\beta$-cell function, insulin resistance, and OCN levels in overweight men.

Methods: In this study, 33 overweight, young men [Body mass index (BMI): 29.32 \pm 0.75 and age range of 31.50 \pm 2.23 ] were randomly divided into control $(n=11)$, aerobic exercise $(n=11)$, and resistance exercise $(n=11)$ groups. Participants of the exercise group were on the 8-week supervised exercise training program for three sessions per week. Weight, body fat percentage, and BMI were analyzed, and then OCN, insulin, and the homeostasis model assessment of insulin resistance (HOMA-IR) were assessed from fasting blood samples before and after the 8-week exercise program. Finally, data were analyzed by $t$ test and analysis of covariance (ANCOVA).

Results: Based on the results, BMI and body weight, insulin, glucose, and HOMA-IR reduced following the exercise $(P<0.05)$ whereas serum OCN significantly increased in aerobic exercise $(P=0.001)$ and resistance exercise $(P=0.000)$ groups. There were no significant changes in $\beta$-cell function in aerobic exercise $(P=0.512)$ and resistance exercise $(P=0.16)$ groups. Pearson correlation analysis demonstrated that OCN levels were not correlated with HOMA-IR $(P=0.743)$ and insulin levels $(P=0.143)$. However, OCN was positively associated with the homeostasis model assessment of b-cell function $(P=0.014)$ and glucose $(P=0.025)$.

Conclusion: The results of the present study confirmed that aerobic and resistance exercises cause some changes in body weight and $\mathrm{BMI}$, as well as the OCN and HOMA-IR. Nonetheless, changes in OCN levels were not predictors of changes in insulin secretion from pancreatic beta cells.

Keywords: Osteocalcin, HOMA-IR, $\beta$-cell function, Exercise, Overweight
\end{abstract}

Received: 16 October 2019, Accepted: 15 February 2021, ePublished: 29 September 2021

\section{Introduction}

The skeletal system is an endocrine organ that plays a role in regulating energy and glucose metabolism. The bone cells, regulates glucose metabolism and causing to insulin secretion from $\beta$-cell and decrease insulin resistance (1). In recent years, the role of the skeleton as an endocrine organ has been increasingly investigated and confirmed, particularly its cross-talk relation with glucose and lipid metabolism. These studies have shown that osteocalcin $(\mathrm{OCN})$ is a new bone-derived hormone and regulator of glucose hemostasis (1). This extracellular matrix non-collagen protein, which is also known as gammacarboxy-glutamic acid (B-GLA) protein, is associated with bone mineralization and calcium ion homeostasis. The circulating OCN has an effect on pancreatic $\beta$-cell proliferation. The non-carboxylic forms of OCN are hormonally active and can stimulate insulin secretion from pancreatic $\beta$-cells and increase insulin sensitivity in muscle and adipose tissues (2). OCN, which is mainly expressed by osteoblasts in bone tissues, can improve insulin sensitivity and insulin secretion through pancreatic $\beta$-cells and their proliferation (3). Researchers showed that changes in insulin signaling in osteoblasts improve glucose metabolism in mice that had been fed a high-fat diet. In addition, insulin resistance in osteoblasts causes a decrease in OCN activity, and therefore, reducing insulin sensitivity in adipose tissues and skeletal muscles (4). These findings heavily suggested the role of OCN in regulating glucose metabolism. The metabolic effects of insulin may be mediated by the bone through OCN release. Finally, there might be other bone-derived mediators through which the bone regulates glucose metabolism and body fat tissues, including the $\mathrm{Wnt} / \beta$-catenin signaling pathway in osteoblasts $(5,6)$.

It has been reported that the improved insulin sensitivity with exercise is related to OCN changes (7). OCN levels and insulin sensitivity were related to a group of obese women who were subjected to intervention with the hypocaloric diet and physical activity for 16 weeks. The results represented that physical activity provided a

(C) 2021 The Author(s); Published by Shahrekord University of Medical Sciences. This is an open-access article distributed under the terms of the Creative Commons Attribution License (http://creativecommons.org/licenses/by/4.0), which permits unrestricted use, distribution, and reproduction in any medium, provided the original work is properly cited. 
better response in elevating the serum OCN than the diet alone, even with a lower weight loss in the former group. Reduced visceral fat was the best independent predictor of changes in OCN levels, which was positively related to insulin sensitivity, suggesting that exercise training improves insulin sensitivity by increasing OCN levels (2). Resistance training (RT) decreased bone resorption markers and unchanged bone formation markers whereas endurance running typically raised the makers of both bone formation and bone resorption (8).

Fernandez et al found that RT causes an improvement in the OCN level in obese women and indicated that OCN could be an important regulator of insulin sensitivity by the bone (9). According to Chahla et al, all participants in the exercise program (from one month to one year running) demonstrated a significant increase in OCN. It seems that exercise increases osteoblast differentiation by increasing mechanical load on the bone, which may increase the overall production of $\mathrm{OCN}$, the osteoblastderived protein (10). However, Abseyi et al reported no significant association between insulin resistance, metabolic syndrome parameters, and OCN levels in obese subjects (11). The results of another study investigating the short-time effects (up to 32 hours) of moderate exercise in healthy male sedentary volunteers, aged 20-35 years represented an increase in bone resorption markers 32 hours after moderate exercise without a significant effect on bone formation markers (12). Considering the important role of OCN in energy metabolism, particularly glucose, some researchers suggested that OCN is a marker for metabolic risk and predictive of type 2 diabetes in addition to being a marker of bone metabolism. In this case, García-Martín et al, studying nondiabetic postmenopausal women, revealed that a serum OCN level lower than $13.5 \mathrm{ng} / \mathrm{mL}$ could be a risk of diabetes mellitus (13). In general, the controversial findings of the effect of different exercises on bone metabolism showed that several factors such as type of exercise activity (e.g., weight-bearing), age, and gender may affect the response of bone metabolism indices to the exercises (14).

This study examined whether different exercises are associated with increasing OCN levels in overweight men. It was hypothesized whether aerobic or resistance exercise training would cause increased secretion of OCN from osteoblast and insulin from $\beta$-cell. If the hypothesis is confirmed, this observation would further advance the understanding of the relationship between OCN, insulin, insulin resistance, and physical activity.

\section{Materials and Methods}

\section{Study design}

This semi-experimental study including 8-week exercise training with three groups was conducted between November 2018 and February 2019 at the Department of Physical Education in the Islamic Azad University of Rasht.

\section{Participants}

Thirty-three overweight young males (28-35 years), who had a body mass index (BMI) of $29.32 \pm 0.75 \mathrm{~kg} / \mathrm{m}^{2}$ and were interested in losing weight and participating in an exercise program, were considered for the study. No subject was suffering from systemic diseases, infections, and physical disabilities so that no to be able to undergo aerobic and resistance exercise. Further, none of them had a history of smoking, experiences of exercise training (in the previous 12 months), and medications for metabolic diseases. The sample size was calculated by considering analysis of covariance (ANCOVA), three groups, type I error of $5 \%$, power of the statistical test of $80 \%$, and effect size of 0.5 and was measured using $\mathrm{G}^{\star}$ Power software. Using a statistical power of 0.80 , an effect size of 0.5 , and an alpha value of $0.05,40$ individuals (13 per group) were selected while considering the potential dropouts of $15 \%$ (15).

Using a computer-generated random number sequence, the participants were randomized by block allocation with a block size of 6 by a research assistant who was not involved in this research. After baseline assessments, a total of 33 eligible subjects $\left(29<\mathrm{BMI}>30 \mathrm{~kg} / \mathrm{m}^{2}\right)$ were randomized into control, aerobic training (AT), and RT groups each containing 11 cases.

\section{Training programs}

The participants in the training groups were prescribed RT and AT. Exercise programs consisted of three sessions a week for 8 weeks, and each exercise session lasted 60 minutes. In each exercise session, participants were trained to warm up for 10 minutes, and then they received 30-40 minutes of main training. Finally, they were trained to cool down for 10 minutes.

The RT protocol included five exercises per session targeting major muscle groups of the body. The performed exercises were barbell bench press, wide grip lateral pull down, barbell back squat, machine leg press, and unilateral machine leg extension. Three sets in each day with 8-12 repetitions with 50\%-75\% $1 \mathrm{RM}$ started, and the resting time between each two sets, as well as between the training sessions were 60-90 seconds and 48 hours, respectively (16). The aerobic exercise comprised of a treadmill running at $60-85 \% \mathrm{HR}_{\max }$. to burn approximately $500 \mathrm{kcal}$ per session (17). Aerobic and RT details are presented in Tables 1 and 2.

To prevent confounding, subjects were instructed to refrain from performing any additional resistance-type or high-intensity AT during the study.

\section{Anthropometric evaluation}

Body weight and height $\left(\mathrm{kg} / \mathrm{m}^{2}\right)$ were measured by the SECA height device (Japan Technology, $0.1 \mathrm{~cm}$ ) in the morning with subjects wearing light clothing and barefoot, and their BMIs were calculated by the weight/height ${ }^{2}(18)$. 
Table 1. Aerobic training details

\begin{tabular}{|c|c|c|c|c|c|}
\hline \multirow{2}{*}{$\begin{array}{c}\text { Week 1-2 } \\
\text { Intensity } \\
\left(\mathrm{HR}_{\max }\right)\end{array}$} & \multirow[b]{2}{*}{ Duration } & \multicolumn{2}{|c|}{ Week 3-6 } & \multicolumn{2}{|c|}{ Week 6-8 } \\
\hline & & $\begin{array}{l}\text { Intensity } \\
\left(\mathrm{HR}_{\max }\right)\end{array}$ & Duration & $\begin{array}{c}\text { Intensity } \\
\left(\mathrm{HR}_{\max }\right)\end{array}$ & Duration \\
\hline$\% 65$ & 25 minutes & $\% 65-\% 75$ & 35 minutes & \%75- \%85 & 40 minutes \\
\hline
\end{tabular}

Table 2. Resistance training details

\begin{tabular}{|c|c|c|c|c|c|c|c|}
\hline \multicolumn{2}{|l|}{ Week 1-2 } & \multicolumn{2}{|c|}{ Week 3-4 } & \multicolumn{2}{|c|}{ Week 5-6 } & \multicolumn{2}{|c|}{ Week 7-8 } \\
\hline $\begin{array}{l}\text { Intensity } \\
\text { (1 RM) }\end{array}$ & 3 periods & $\begin{array}{c}\text { Intensity } \\
(1 \mathrm{RM})\end{array}$ & 3 periods & $\begin{array}{c}\text { Intensity } \\
(1 \mathrm{RM})\end{array}$ & 3 periods & $\begin{array}{c}\text { Intensity } \\
(1 \mathrm{RM})\end{array}$ & 3 periods \\
\hline$\% 50$ & 10 repeats & $\% 60$ & $10-12$ repeats & $\% 65$ & 10 repeats & $\% 75$ & $10-12$ repeats \\
\hline
\end{tabular}

\section{Biochemical analyses}

Blood samples were collected from all participants after 12 hours of fasting between 8:00 and 10:00 a.m. to minimize hormonal rhythmicity at the start of the study and 2 days after the termination of the study. Samples were immediately centrifuged at $3000 \mathrm{~g}$ for 10 minutes at $4^{\circ} \mathrm{C}$, and the serum samples were stored at $-70^{\circ} \mathrm{C}$ until analysis. Fasting blood glucose (FBG) was quantified using an Autoanalyzer device (Technicon, RA1000, USA) with Pars Azmoon kits (Karaj, Iran). Fasting plasma insulin (FPI) was also measured by Electrochemiluminescence immunoassay (kits of liaison, England).

The serum levels of OCN were determined by the Human OCN ELISA Kit (Hangzhou Eastbiopharm Company, LTD., China) with a sensitivity of $0.026 \mathrm{ng} / \mathrm{mL}$.

Insulin resistance and $\beta$-cell function were estimated by the homeostasis model assessment of insulin resistance (HOMA) as follows (18):

HOMA-IR $=$ FBG $(\mathrm{mg} / \mathrm{dL}) \times$ FPI $(\mu \mathrm{u} / \mathrm{mL}) / 405$

HOMA-B $=360 \times$ FPI $(\mu \mathrm{u} / \mathrm{mL}) /$ FBG $(\mathrm{mg} / \mathrm{dL})-63$

\section{Nutrition}

To avoid the potential dietary confounding of the results, subjects were advised to maintain their customary nutritional regimen and avoid taking any supplements that affected the results. Dietary adherence was assessed by self-reported food records using a nutritional tracking application (http://www.myfitnesspal.com), which was collected each week during the study. Subjects were instructed on how to properly record all food items and their respective portion sizes consumed for the designated period of interest. Each item of food was individually entered into the program, and the program provided relevant information as to total energy consumption, as well as the amount of energy derived from proteins, fats, and carbohydrates for each time period analyzed. All participants used the same nutrition program designed by the nutritionist. Nutrition details are provided in Table 3.

\section{Statistical analyses}

All data were analyzed by Statistical Package for Social Sciences software (SPSS, 25.0 K; IBM, USA) and data were presented as the mean \pm standard deviation. Pearson's bivariate correlation analysis and paired t-test were used to evaluate the associations among the variables and examine the differences in the baseline and follow-up variables within the group, respectively. The variables among the groups were compared using the ANCOVA test to conservatively locate significant differences. Finally, the Kolmogorov-Smirnov test was performed for variables that did not exhibit a normal distribution, and the levels of statistical significance were set at $P<0.05$.

\section{Results}

Thirty-three overweight subjects participated in this study, and those within the age range of 28-35 with a mean age of $31.50 \pm 2.23$ years were enrolled in this study. Table 4 summarizes data related to body weight, body composition, OCN, insulin, HOMA-IR, and $\beta$-cell levels. The results revealed no significant differences in body composition and OCN levels between the three groups in the pretest.

However, there were significant differences between control and exercise training groups with respect to BMI changes after 8 weeks of intervention (A, $\mathrm{Ex}, \quad$ pre $=29.33 \pm 0.75$, post $=27.34 \pm 0.99$ and $\mathrm{R}, \mathrm{Ex}$, pre $=28.76 \pm 1.05$, post $=28.53 \pm 1.05$ ). The BMI decreased in the exercise groups after training. The changes in body fat percentage between the three groups were also significantly different so that body fat decreased at the 8-week period in both exercise groups (A $\mathrm{Ex}$, pre $=20.52 \pm 3.68$, post $=19.34 \pm 3.45$ and $\mathrm{R}, \mathrm{Ex}$, pre $=18.63 \pm 1.49$, post $=18.12 \pm 1.37$ ). Based on the results, significant differences were found in the changes of OCN, insulin, HOMA-IR, and $\beta$-cell function levels between the two intervention groups and the control group. This finding demonstrated the effects of exercise on both experimental groups compared with control groups

Table 3. Nutrition details of all subjects

\begin{tabular}{lccc}
\hline Variable & Saturday & Tuesday & P value \\
\hline Energy (kcal) & $2204.00 \pm 122.9$ & $2212.00 \pm 113.8$ & 0.48 \\
Protein $(\mathrm{g} . \mathrm{kg})$ & $14.6 \pm 1.5$ & $14.3 \pm 3.2$ & 0.84 \\
Carbohydrates (kcal) & $52.9 \pm 4.2$ & $52.2 \pm 3.85$ & 0.56 \\
Fat $(\% \mathrm{kcal})$ & $32.5 \pm 3.9$ & $33.5 \pm 3.13$ & 0.39 \\
\hline
\end{tabular}




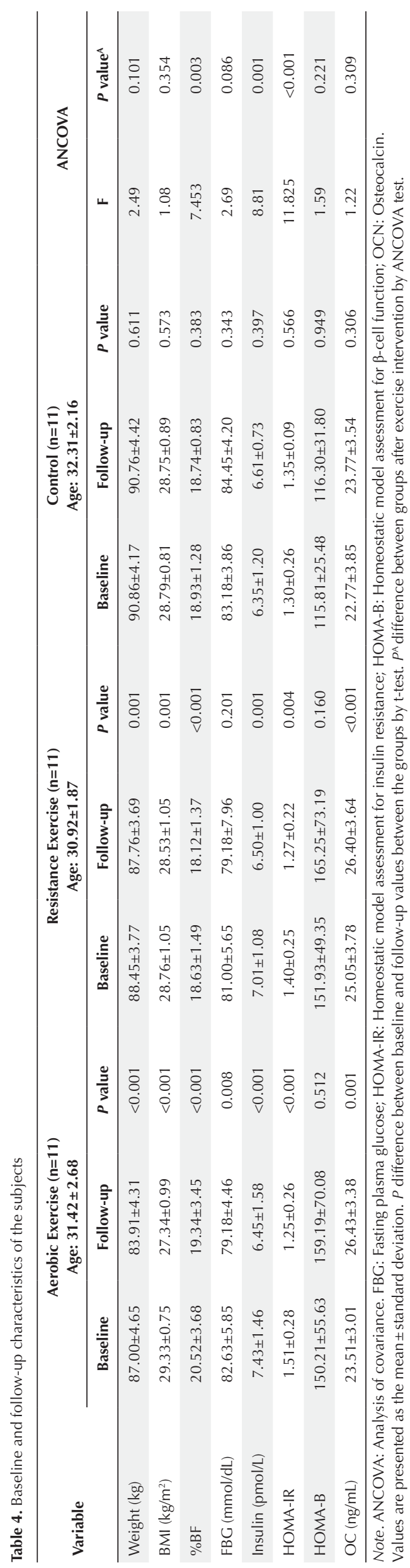


regarding changing $\mathrm{OCN}$, insulin resistance, and insulin secretion from $\beta$-cells.

Body composition and biochemical parameters decreased in both two Ex groups (RT and AT). Specifically, weight, BMI, and BF\% demonstrated a significant decrease in the two Ex groups (RT and AT). Additionally, Ex groups showed a reduction in FBS (AT, $P=0.008$ and RT, $P=0.201$ ), FPI (AT, $P=0.000$ and RT, $P=0.001$ ), and HOMA-IR (AT, $P=0.000$ and RT, $P=0.004$ ) while a significant increase in OCN (AT, $P=0.001$ and RT, $P=0.000)$ compared with the control group. However, no significant changes were observed in $\beta$-cell function in the two exercise groups (AT, $P=0.512$ and RT, $P=0.16$ ). There were no significant differences regarding FBS in resistance Ex groups. Considering the difference in the mean on a comparison between the groups, the results showed no significant difference between exercise groups. The correlations between changes in body composition parameters and circulating levels of OCN were evaluated as well. Pearson correlation revealed no significant correlation between the changes in BMI, total fat (\%), and weight with changes in the OCN level in all subjects. Moreover, Pearson correlation represented that changes in biochemical parameters had no significant correlation with changes in the OCN level in all subjects (Table 5).

\section{Correlation Between OCN and Insulin Resistance, $\beta$-cell Function, and Insulin}

Based on Pearson correlation results (Table 5), OCN levels demonstrated a negative correlation with FBG $(\mathrm{r}=-0.480, P=0.005)$. Conversely, the results showed a positive relation between OCN and HOMA-B $(r=0.469$, $P=0.006)$. Eventually, no significant relationships were observed between OCN and insulin and HOMA-IR.

\section{Discussion}

In general, the findings of the present study demonstrated that 8-week exercise training led to a decrease in body weight, BMI while improving insulin resistance and increasing the serum OCN level in overweight young males. Although the effect of AT on increasing OCN and insulin secretion was higher in comparison with the RT group, these differences were not statistically significant.

The bone regeneration process is significantly affected

Table 5. Linear Associations Among Baseline OCNs and the Measures of Blood Biochemical Parameters

\begin{tabular}{lcc}
\hline & \multicolumn{2}{c}{ Osteocalcin } \\
\cline { 2 - 3 } & $\mathbf{R}$ & $\boldsymbol{P}$ \\
\hline FBG & -0.480 & $0.005^{*}$ \\
Insulin & 0.261 & 0.143 \\
HOMA-B & 0.469 & $0.006^{*}$ \\
HOMA-IR & 0.059 & 0.743 \\
\hline
\end{tabular}

Note. OCN: Osteocalcin; FBG: Fasting blood glucose; HOMA-B: Homeostatic model assessment for $\beta$-cell function; HOMA-IR: Homeostatic model assessment for insulin resistance; ${ }^{*} P<0.05$; $P$ significant correlations. by mechanical loads. Previous research revealed that mechanical pressures by the weight-bearing exercise were the most important factors affecting the formation of new bones and increased bone mineral density (19).

It seems that the decarboxylation of $\mathrm{OCN}$ is a process that occurs outside the osteoblasts during bone resorption by osteoclasts. The low $\mathrm{pH}$ of osteoclasts (4.5) in the resorption lacuna, decarboxylates at least one of the available Gla residues in $\mathrm{OCN}$, thus allowing the release of bioactive OCN. Insulin and OCN in a feedforward loop control glucose metabolism. Indeed, insulin signaling in osteoblasts, through the inhibition of the transcription factor FOXO1, reduced the expression of osteoprotegerin, which is a negative regulator of bone resorption, thereby inducing increased osteoclastic activity and the release of bioactive OCN (20).

In the present study, the serum concentrations of OCN, insulin, and insulin resistance were statistically different between the two experimental and control groups. It was shown that OCN levels significantly increased in the experimental groups after 8-week exercise intervention while a reduction was observed in glucose, insulin, and insulin resistance in the two exercise groups.

Few studies have currently examined how OCN contributes to the exercise-induced improvement in metabolic parameters. Yu et al reported that moderate aerobic exercise significantly increased OCN levels in obese men. They further found that there is a significant correlation between the increase in OCN levels and insulin resistance (21). Similarly, Levinger et al concluded that the acute aerobic exercise significantly increased undercarboxilated Osteocalcin (ucOC) while the acute resistance exercise did only marginally among middleaged obese subjects with type 2 diabetes mellitus (22). Another recent study by Ghorbanian et al demonstrated that the 8-week aerobic exercise significantly increased OCN while significantly decreasing insulin resistance levels in obese postmenopausal women with type 2 diabetes mellitus (23). As one of the main mechanisms, the exercise can increase the serum level of OCN, because more activity of bone cells and responses to mechanical stresses by exercise training result in more OCN secretion by these cells. The latter affects the hemostasis of energy metabolism during physical activity in the body. Given that bone is recently known as an active metabolic tissue, signals from insulin and glucose changes lead to more bone activity and stimulate OCN secretion during physical activity (2).

In their study, Turky et al found that the combined exercise training (aerobic + resistance) on the overweight women cause a decrease in OCN levels followed by exercise training (24), which contradicts our findings. Regarding the relationship between exercise intensity and OCN levels, the intensity and duration of the exercise program are among the major contradictory factors in the findings of the study. Likewise, OCN is considered as a hormone that is involved in the regulation of energy 
metabolism. The conditions for controlling the nutritional status and the genetic factors of the subjects may be other contradictions between the findings of the present study and those of previous research (25).

In the present study, insulin, glucose, and insulin resistance significantly decreased following 8 weeks of aerobic and resistance exercise training. Contrarily, Azerbaijani et al reported that three types of exercise training (i.e., aerobic, resistance, and combined training) in middle-aged inactive men caused a decrease in glucose and insulin levels (26). In their study on inactive men, Nouri et al demonstrated that various exercises reduced insulin and glucose levels, as well as weight and body fat percentage and the BMI while increasing the levels of insulin sensitivity in different individuals of different genders (27).

Long-term exercises can increase glucose transporters to muscle cells and insulin receptor substrates, and an increase in muscle mass (more than $75 \%$ of glucose utilization due to insulin-induced muscle tissue stimulation) causes the body response to insulin, increasing insulin sensitivity and preventing obesity and its subsequent complications. Aerobic exercises are shown to increase insulin function by reducing intracellular triglyceride accumulations whereas increasing the oxidation of fatty acids (28). Contrarily, Attarzadeh et al found that aerobic exercise in obese women failed to significantly change glucose and insulin levels. According to the researchers, the intensity and duration of the exercise are the main causes of the contradiction between the findings in the studies (29).

Another object of this study was to find a correlation between OCN and insulin resistance and $\beta$-cell function in overweight men. Based on the results, significant correlations were observed between increased OCN levels and glucose and $\beta$-cell function.

$\mathrm{OCN}$ is produced from osteoblasts and is activated at a low $\mathrm{pH}$ of the resorption cavity. Insulin may be regulated by OCN bioactivity through insulin receptors on osteoblasts (30). Conversely, OCN has been reported to impact $\beta$-cell proliferation, insulin secretion, and insulin sensitivity (31) through GPCR6a receptors in $\beta$-cells (32). Previous studies reported a significant correlation between OCN and glucose levels in overweight men $(33,34)$. These findings suggest that the bioactive form of $\mathrm{OCN}$ is released into the bloodstream that mainly affects two ways of glucose metabolism. First, OCN directly affects the $\beta$-cell function by binding to receptor GPRC6A and increasing $\beta$-cell proliferation, as well as synthesis and insulin secretion. Second, OCN improves insulin sensitivity and energy expenditure via multiple mechanisms. OCN stimulates energy expenditure by increasing mitochondrial biogenesis in the muscle and regulates gene expression implicated in energy consumption in adipose tissues and skeletal muscles. OCN also affects insulin sensitivity possibly by increasing adiponectin expression in adipose tissues while reducing lipid accumulations and inflammation in the steatotic liver. A direct effect of
OCN as an insulin-sensitizing hormone is speculative and requires further evaluation (20).

\section{Conclusion}

The results of this study showed that both AT and RT significantly increased the serum levels of OCN and insulin sensitivity while decreasing insulin and insulin resistance. Nonetheless, they made no significant changes in $\beta$-cell function in overweight young men. However, no significant difference was found between AT and RT. Participation in the exercise program seems to improve body composition while reducing adipose tissue and weight. The findings of previous studies confirmed the association of OCN with glucose metabolism. Thus, AT and RT both caused changes in OCN secretions in the energy metabolism and body weight by increasing mechanical load on bone mass. Therefore, the change in OCN can be an important factor in increasing bone mass and weight loss in overweight and obese people.

\section{Conflict of Interests}

The authors declared that they have no conflict of interests.

\section{Ethical Approval}

This study was approved by the Research Ethics Committees of Islamic Azad University of Rasht, Rasht, Iran (ethical code of IR.IAU.RASHT.REC.1396.124). Additionally, It was registered in the Iranian Registry of Clinical Trials website (identifier: IRCT20180226038876N1; https://www.irct.ir/trial/32387) All participants provided written informed consent before participating in the study.

Authors' Contributions

M. R. designed and performed the experiments and acquired data, and then designed the study, interpreted the data, and wrote the manuscript with the help of A. E. and F. R. All authors reviewed the manuscript and provided the final approval of the version for publication. M. R. is the guarantor of this work, has full access to all the data in the study, and takes responsibility for the integrity of the data and the accuracy of the analysis.

\section{Funding/Support}

None.

\section{Acknowledgments}

This article is part of a Ph.D. thesis (under number 11721404961011) conducted in January 2019 in the Department of Physical Education of Islamic Azad University, Rasht. Special thanks go to the efforts of the supervisors, consultants, and all loved ones who have attempted to do this study.

\section{References}

1. Yeap BB. Osteocalcin: an endocrine link between bone and glucose metabolism. Expert Rev Endocrinol Metab. 2011;6(2):177-85. doi: 10.1586/eem.11.7.

2. Zanatta LC, Boguszewski CL, Borba VZ, Kulak CA. Osteocalcin, energy and glucose metabolism. Arq Bras Endocrinol Metabol. 2014;58(5):444-51. doi: 10.1590/00042730000003333.

3. Lenora J, Ivaska KK, Obrant KJ, Gerdhem P. Prediction 
of bone loss using biochemical markers of bone turnover. Osteoporos Int. 2007;18(9):1297-305. doi: 10.1007/s00198007-0379-z.

4. Wei J, Ferron M, Clarke CJ, Hannun YA, Jiang H, Blaner WS, et al. Bone-specific insulin resistance disrupts whole-body glucose homeostasis via decreased osteocalcin activation. J Clin Invest. 2014;124(4):1-13. doi: 10.1172/jci72323.

5. Fulzele K, Lai F, Dedic C, Saini V, Uda Y, Shi C, et al. Osteocyte-secreted Wnt signaling inhibitor sclerostin contributes to beige adipogenesis in peripheral fat depots. J Bone Miner Res. 2017;32(2):373-84. doi: 10.1002/ jbmr.3001.

6. Yao Q, Yu C, Zhang X, Zhang K, Guo J, Song L. Wnt/ $\beta$ catenin signaling in osteoblasts regulates global energy metabolism. Bone. 2017;97:175-83. doi: 10.1016/j. bone.2017.01.028.

7. Campos RM, de Mello MT, Tock L, Silva PL, Masquio DC, de Piano A, et al. Aerobic plus resistance training improves bone metabolism and inflammation in adolescents who are obese. J Strength Cond Res. 2014;28(3):758-66. doi: 10.1519/JSC.0b013e3182a996df.

8. Mera P, Laue K, Ferron M, Confavreux C, Wei J, Galán-Díez $\mathrm{M}$, et al. Osteocalcin signaling in myofibers is necessary and sufficient for optimum adaptation to exercise. Cell Metab. 2016;23(6):1078-92. doi: 10.1016/j.cmet.2016.05.004.

9. Fernández-Real JM, Izquierdo $M$, Ortega $F$, Gorostiaga E, Gómez-Ambrosi J, Moreno-Navarrete JM, et al. The relationship of serum osteocalcin concentration to insulin secretion, sensitivity, and disposal with hypocaloric diet and resistance training. J Clin Endocrinol Metab. 2009;94(1):237-45. doi: 10.1210/jc.2008-0270.

10. Chahla SE, Frohnert BI, Thomas W, Kelly AS, Nathan BM, Polgreen LE. Higher daily physical activity is associated with higher osteocalcin levels in adolescents. Prev Med Rep. 2015;2:568-71. doi: 10.1016/j.pmedr.2015.06.017.

11. Abseyi N, Şıklar Z, Berberoğlu M, Hacıhamdioğlu B, Savaş Erdeve Ş, Öçal G. Relationships between osteocalcin, glucose metabolism, and adiponectin in obese children: Is there crosstalk between bone tissue and glucose metabolism? J Clin Res Pediatr Endocrinol. 2012;4(4):1828. doi: $10.4274 /$ jcrpe. 831 .

12. Welsh L, Rutherford OM, James I, Crowley C, Comer M, Wolman R. The acute effects of exercise on bone turnover. Int J Sports Med. 1997;18(4):247-51. doi: 10.1055/s-2007972628.

13. García-Martín A, Cortés-Berdonces M, Luque-Fernández I, Rozas-Moreno P, Quesada-Charneco M, Muñoz-Torres M. Osteocalcin as a marker of metabolic risk in healthy postmenopausal women. Menopause. 2011;18(5):537-41. doi: 10.1097/gme.0b013e3181f8565e.

14. Vainionpää A, Korpelainen R, Väänänen HK, Haapalahti J, Jämsä T, Leppäluoto J. Effect of impact exercise on bone metabolism. Osteoporos Int. 2009;20(10):1725-33. doi: 10.1007/s00198-009-0881-6.

15. Banitalebi E, Tavasoli Z, Jafari T, Mardanian M. The effect of elastic resistance band training with green coffee supplementation on novel hepatic steatosis biomarkers in obese women: A randomized controlled trial. J.skums. 2020;22(4):187-94. 10.34172/jsums.2020.30

16. Soori R, Ravasi A, Ranjbar K. The comparison of between endurance and resistance training on vaspin and adiponectin in obese middle-age men. S Physiol, 2014;
5(20), 97-114.

17. Asad M. Effect of 8 weeks aerobic, resistance and concurrent training on cholestrol, LDL, HDL and cardiovascular fitness in obesity male. Applied Research in Sport Management. 2013;1(3):57-64. [Persian].

18. Giudici KVP, Fisberg RM, Marchioni DML, Peters BSE, Martini LA. Crosstalk between bone and fat tissue: associations between vitamin $\mathrm{D}$, osteocalcin, adipokines, and markers of glucose metabolism among adolescents. J Am Coll Nutr. 2017;36(4):273-80. doi: 10.1080/07315724.2016.1274923.

19. Abbaszadeh surati $\mathrm{H}$, Abrahim KH, Nikbakht HA. The effect of 16-week selective aerobic exercise on serum osteopontin, and osteocalcin in sedentary middle-aged women. Journal of Sport and Exercise Physiology. 2011;5(2):778-84.

20. Mera P, Ferron M, Mosialou I. Regulation of energy metabolism by bone-derived hormones. Cold Spring Harb Perspect Med. 2018;8(6). doi: 10.1101/cshperspect.a031666.

21. Kim YS, Nam JS, Yeo DW, Kim KR, Suh SH, Ahn CW. The effects of aerobic exercise training on serum osteocalcin, adipocytokines and insulin resistance on obese young males. Clin Endocrinol (Oxf). 2015;82(5):686-94. doi: 10.1111/cen.12601.

22. Levinger I, Jerums G, Stepto NK, Parker L, Serpiello FR, McConell GK, et al. The effect of acute exercise on undercarboxylated osteocalcin and insulin sensitivity in obese men. J Bone Miner Res. 2014;29(12):2571-6. doi: 10.1002/jbmr.2285.

23. Ghorbanian B, Barani A. Study the effect of exercise on bone markers, glycemic and anthropometric indices in postmenopausal women with diabetes. J Arak Uni Med Sci. 2017;20(1):107-17. [Persian].

24. Turky KT. Effects of combined exercise training on osteocalcin, body weight, and inflammatory markers among pre-obese women. Med J Cairo Univ. 2015;83(2):233-8.

25. Rached MT, Kode A, Xu L, Yoshikawa Y, Paik JH, Depinho RA, et al. FoxO1 is a positive regulator of bone formation by favoring protein synthesis and resistance to oxidative stress in osteoblasts. Cell Metab. 2010;11(2):147-60. doi: 10.1016/j.cmet.2010.01.001.

26. Azarbayjani MA, Abedi B. Comparison of aerobic, resistance and concurrent exercise on lipid profiles and adiponectin in sedentary men. Knowledge And Health. 2012;7(1):32-8. [Persian].

27. Nouri Y, Rahmani nia F, Mirzaie B, Arazi H. The effect of resistance and endurance training on resting metabolic rate and body composition in sedentary males. J Adv Med Biomed Res. 2013;21(89):51-63. [Persian].

28. Azali Alamdari K, khalafi m, Ghorbanian B. Effect of aerobic training on serum adiponectin and Ctrp-3 in males with metabolic syndrome. Iran J Endocrinol Metab. 2017;18(5):368-77. [Persian].

29. Attarzadeh Hosseini R, Sardar M A, Taghavi M, Ayaz Khosh Hava F. The effects of an aerobic exercise program on LH, FSH, TST and DHEA levels in obese women with polycystic ovary syndrome. Iran J Endocrinol Metab. 2012;14(1):39-46. [Persian].

30. Wei J, Karsenty G. An overview of the metabolic functions of osteocalcin. Rev Endocr Metab Disord. 2015;16(2):93-8. doi: 10.1007/s11154-014-9307-7.

31. Nordström A, Högström M, Nordström P. Effects of different types of weight-bearing loading on bone mass 
and size in young males: a longitudinal study. Bone. 2008;42(3):565-71. doi: 10.1016/j.bone.2007.11.012.

32. Gastaldelli A, Gaggini M, DeFronzo RA. Role of adipose tissue insulin resistance in the natural history of type 2 diabetes: results from the San Antonio Metabolism Study. Diabetes. 2017;66(4):815-22. doi: 10.2337/db16-1167.

33. Kanazawa I, Yamaguchi T, Yamamoto M, Yamauchi M, Kurioka S, Yano S, et al. Serum osteocalcin level is associated with glucose metabolism and atherosclerosis parameters in type 2 diabetes mellitus. J Clin Endocrinol Metab. 2009;94(1):45-9. doi: 10.1210/jc.2008-1455.

34. Iki M, Tamaki J, Fujita Y, Kouda K, Yura A, Kadowaki E, et al. Serum undercarboxylated osteocalcin levels are inversely associated with glycemic status and insulin resistance in an elderly Japanese male population: Fujiwara-kyo Osteoporosis Risk in Men (FORMEN) study. Osteoporos Int. 2012;23(2):761-70. doi: 10.1007/s00198-011-1600-7. 